\title{
Antioxidative and Anti-Inflammatory Activities of Galloyl Derivatives and Antidiabetic Activities of Acer ginnala
}

\author{
Kwan Hee Park, Kyu Hyeong Yoon, Jun Yin, Thi Tam Le, Hye Sin Ahn, \\ Seong Hye Yoon, and Min Won Lee
}

Laboratory of Pharmacognosy and Natural Product Derived Medicine, College of Pharmacy, Chung-Ang University, Seoul 156-756, Republic of Korea

Correspondence should be addressed to Min Won Lee; mwlee@cau.ac.kr

Received 11 November 2016; Revised 24 January 2017; Accepted 8 February 2017; Published 2 March 2017

Academic Editor: G. K. Jayaprakasha

Copyright (C) 2017 Kwan Hee Park et al. This is an open access article distributed under the Creative Commons Attribution License, which permits unrestricted use, distribution, and reproduction in any medium, provided the original work is properly cited.

Chromatographic isolation of the $80 \% \mathrm{MeOH}$ extract of Acer ginnala (AG) yielded seven galloyl derivatives: gallic acid (1), ginnalin B (2), acertannin (3), maplexin D (4), maplexin E (5), quercetin-3-O-(2" -galloyl)- $\alpha$-L-rhamnopyranoside (6), and kaempferol-3$O$ - $\left(2^{\prime \prime}\right.$-galloyl $)-\alpha$-L-rhamnopyranoside (7). This is the first study to report the isolation of compounds 4 and 5 from AG. Galloyl derivatives 3-7 exhibited potent radical scavenging activities, with $\mathbf{5}$ and $\mathbf{7}$ showing particularly strong inhibitory activities against nitric oxide production in lipopolysaccharides- (LPS-) stimulated RAW264.7 cells. In addition, oral administration of AG extract $(500 \mathrm{mg} / \mathrm{kg}$ b.w.) improved symptoms of hyperglycemia and blunted the increases in serum GOT/GPT levels in a rat model of streptozotocin-induced diabetes. These results suggest that galloyl derivatives (1-7) are antioxidant and anti-inflammatory agents and that AG extract has potential as a functional material or novel herbal medicine for treating diabetes mellitus.

\section{Introduction}

Diabetes, one of the deathful diseases, is well known worldwide. According to the report of WHO, the number of people with diabetes in 1980 (108 million) has risen to 422 million in 2014 [1]. It is reported that diabetes will be the 7 th leading cause of death in 2030 [2]. Between 2 types of diabetes, type 2 diabetes results from ineffective use of insulin, and the majority of people with diabtes worldwide are this type [3]. Oxidative stress is one of the pathways that could induce diabetes, because the high glucose level could simulate free radical production to make imbalance ROS [4]. And it is reported that inflammation is associated with the development of type 2 diabetes, such as proinflammatory cytokines interleukin-1 beta and tumor necrosis factor-alpha [5]. Thus, the antioxidative and anti-inflammatory activities are important ways to treat diabetes.

The genus Acer (Aceraceae), commonly known as maple, is the largest group of deciduous trees and shrubs. This genus comprises approximately 130 species occupying a significant part of the northern hemisphere [6]. In Northeast Asia, Acer species have been used in pharmaceutical preparations such as decoctions or teas to lower fever, improve eyesight, and protect the liver. In addition, Acer species have been used by the native population of eastern Canada as traditional remedies for treating coughs and eye pain [7]. In the present day, Acer species are commonly used in commercial products in North America.

Acer ginnala (AG), otherwise known as "amur maple," is native to northeastern Asia. In Republic of Korea, AG has been used as a traditional folk medicine for the treatment of eye disease, wound healing, and diarrhea [8]. The crystalline tannin acertannin was first discovered in AG [9]; the structure of this tannin was later fully elucidated as 2,6-di$O$-galloyl-1,5-anhydro-D-glucitol [10]. To date, several gallotannins, flavonoids, and terpenoids have been isolated from AG [11, 12]. Moreover, AG has been reported to demonstrate antioxidative, antibacterial, and antitumor activities $[13,14]$.

The sap of Acer species, that is, maple syrup, is believed to be a suitable sweetener for the management of diabetes mellitus [15]. Recently, AG extract was reported to inhibit rat lens aldose reductase and the formation of advanced glycation products [16]. Furthermore, ginnalin A-C from other 
Acer species $[17,18]$ has been reported to exhibit antihyperglycemic effects in a sucrose-loaded mouse model of diabetes. However, these previous studies used enzymatic tests or the acute glucose tolerance test, which are not sufficient to fully characterize the antidiabetic activity of AG [19]. The aim of this study was to isolate biological compounds from the leaves of AG and to evaluate the hypoglycemic activity of AG in a rat model of streptozotocin- (STZ-) induced diabetes.

\section{Materials and Methods}

2.1. Plant Material. The leaves of AG (1 kg) were collected from the Korea National Arboretum (Pocheon, Republic of Korea) in 2010. A voucher specimen (AG2010) was deposited at the herbarium of the College of Pharmacy, Chung-Ang University (Seoul, Republic of Korea).

2.2. General Procedures. Large-scale chromatographic isolation was performed on a Sephadex LH-20 column (10-25 $\mu \mathrm{m}$, GE Healthcare Bio-Sciences AB, Uppsala, Sweden). Daisogel (40-60 $\mu \mathrm{m}$, Daiso, Osaka, Japan) was used as the stationary phase on a middle pressure liquid chromatography (MPLC) system (Gilson, Seoul, Republic of Korea). The isolation process was monitored by thin layer chromatography (TLC) using a precoated silica gel 60 F254 plate (Merck, Darmstadt, Germany). Spots were detected under UV radiation $(254 \mathrm{~nm})$ and by spraying with $\mathrm{FeCl}_{3}$ and $10 \% \mathrm{H}_{2} \mathrm{SO}_{4}$ or anisaldehyde- $\mathrm{H}_{2} \mathrm{SO}_{4}$, followed by heating. The compounds from the leaves of $A G$ were identified by $1 D\left({ }^{1} \mathrm{H} /{ }^{13} \mathrm{C}\right)$ and 2D (COSY, HSQC, and HMBC) nuclear magnetic resonance (NMR) spectroscopy (Varian, Palo Alto, CA) at Chung-Ang University.

2.3. Extraction and Isolation. The leaves ( $1 \mathrm{~kg})$ of AG were extracted three times with $80 \%$ methanol at room temperature. After removing the methanol under vacuum, $322.5 \mathrm{~g}$ of extract was dissolved in water. The resultant aqueous solution was filtered through Celite 545 (Duksan Pure Chemicals, Ansan, Republic of Korea). Next, $227.5 \mathrm{~g}$ of watersoluble concentrated filtrate was applied to a Sephadex LH20 column $(2 \mathrm{~kg}, 10 \mathrm{~cm} \times 80 \mathrm{~cm})$ equilibrated with distilled water. The column was eluted with a water-methanol gradient system, yielding four fractions. Subfraction 1 (6.4 g) was subjected to Daisogel ODS $(300 \mathrm{~g}, 3 \times 50 \mathrm{~cm})$ and eluted from the MPLC system using a $20-100 \%$ methanol gradient $(5 \mathrm{~mL} / \mathrm{min}, 280 \mathrm{~nm})$ and yielded ginnalin B (2, $25 \mathrm{mg})$. Subfraction $2(79.5 \mathrm{~g})$ was subjected to Daisogel ODS $(300 \mathrm{~g}, 3 \times 50 \mathrm{~cm}$ ) and eluted from the MPLC system using a $20-100 \%$ methanol gradient $(5 \mathrm{~mL} / \mathrm{min}, 280 \mathrm{~nm})$ and yielded gallic acid (1, $25 \mathrm{mg})$ and maplexin E (5, $20 \mathrm{mg})$. Subfraction 4 (43.1 g) was subjected to Daisogel ODS (300 g, $3 \times 50 \mathrm{~cm})$ and eluted from the MPLC system using a 20$100 \%$ methanol gradient $(5 \mathrm{~mL} / \mathrm{min}, 280 \mathrm{~nm})$ and yielded gallic maplexin D $(4,20 \mathrm{mg})$, quercetin-3-O-( $2^{\prime \prime}$-galloyl $)-\alpha$ L-rhamnopyranoside $(6,150 \mathrm{mg})$, and kaempferol-3-O-( $2^{\prime \prime}$ galloyl)- $\alpha$-L-rhamnopyranoside $(7,850 \mathrm{mg})$. Subfraction 3 (100.2 g) was recrystallized, yielding acertannin (3, $80 \mathrm{~g})$.

\subsubsection{Characterization of Compounds Isolated from AG}

Compound 1 [3,4,5-Trihydroxybenzoic Acid, Gallic Acid] $(S 1,2)$

Gray Amorphous Powder. ${ }^{1} \mathrm{H}-\mathrm{NMR}\left(600 \mathrm{MHz}, \mathrm{CD}_{3} \mathrm{OD}\right): \delta$ 7.06 (2H, s, H-2, 6); ${ }^{13} \mathrm{C}-\mathrm{NMR}\left(150 \mathrm{MHz}, \mathrm{CD}_{3} \mathrm{OD}\right): \delta 109.02$ (C-2, C-6), $\delta 120.59$ (C1), $\delta 138.15$ (C-4), $\delta 144.95$ (C-3, 5), $\delta$ $169.00(\mathrm{C}-7)$.

Compound 2 [6-Galloyl-1,5-anhydroglucitol, Ginnalin B] $(S 3,4)$

Gray Amorphous Powder. ${ }^{1} \mathrm{H}-\mathrm{NMR}\left(600 \mathrm{MHz}, \mathrm{CD}_{3} \mathrm{OD}\right): \delta$ $3.01(1 \mathrm{H}, \mathrm{t}, J=11.1 \mathrm{~Hz}, \mathrm{H}-1 \mathrm{~b}) \delta 3.11(2 \mathrm{H}, \mathrm{m}, \mathrm{H}-4,5) \delta 3.28(2 \mathrm{H}$, $\mathrm{m}, \mathrm{H}-\mathrm{la}, 3) \delta 3.71(1 \mathrm{H}, \mathrm{m}, \mathrm{H}-2) \delta 4.13(1 \mathrm{H}, \mathrm{dd}, J=4.5,11.7 \mathrm{~Hz}$, H-6b) $\delta 4.39$ (1H, br d, $J=11.7 \mathrm{~Hz}, \mathrm{H}-6 \mathrm{a}) \delta 6.93\left(2 \mathrm{H}, \mathrm{s}, \mathrm{H}-2^{\prime}\right.$, $\left.6^{\prime}\right) ;{ }^{13} \mathrm{C}-\mathrm{NMR}\left(150 \mathrm{MHz}, \mathrm{CD}_{3} \mathrm{OD}\right): \delta 64.40(\mathrm{C}-6), \delta 69.89(\mathrm{C}-$ $1), \delta 69.99(\mathrm{C}-4), \delta 70.39(\mathrm{C}-2), \delta 78.28(\mathrm{C}-3), \delta 78.65(\mathrm{C}-5)$, $\delta 108.98\left(\mathrm{C}-2^{\prime}, \mathrm{C}-6^{\prime}\right), \delta 119.85\left(\mathrm{Cl}^{\prime}\right), \delta 138.66\left(\mathrm{C}-4^{\prime}\right), \delta 145.73$ $\left(\mathrm{C}-3^{\prime}, 5^{\prime}\right), \delta 166.30\left(\mathrm{C}-7^{\prime}\right)$.

Compound 3 [2,6-Digalloyl-1,5-anhydroglucitol, Acertannin] $(S 5,6)$

Gray Amorphous Powder. ${ }^{1} \mathrm{H}-\mathrm{NMR}$ (600 MHz, $\left.\mathrm{CD}_{3} \mathrm{OD}\right): \delta$ $3.34(1 \mathrm{H}, \mathrm{t}, J=11.1 \mathrm{~Hz}, \mathrm{H}-1 \mathrm{~b}) \delta 3.53(2 \mathrm{H}, \mathrm{m}, \mathrm{H}-4,5) \delta 3.72(1 \mathrm{H}$, $\mathrm{t}, J=9.0 \mathrm{~Hz}, \mathrm{H}-3) \delta 4.10(1 \mathrm{H}, \mathrm{dd}, J=5.5,11.1 \mathrm{~Hz}, \mathrm{H}-1 \mathrm{a}) \delta 4.38$ $(1 \mathrm{H}, \mathrm{dd}, J=4.5,11.7 \mathrm{~Hz}, \mathrm{H}-6 \mathrm{~b}) \delta 4.54(1 \mathrm{H}, \mathrm{br} \mathrm{d}, J=11.7 \mathrm{~Hz}$, H-6a) $\delta 4.90(1 \mathrm{H}, \mathrm{m}, \mathrm{H}-2) \delta 7.09\left(2 \mathrm{H}, \mathrm{s}, \mathrm{H}-2^{\prime}, 6^{\prime}\right) \delta 7.10(2 \mathrm{H}$, s, H-2", $\left.6^{\prime \prime}\right) ;{ }^{13} \mathrm{C}-\mathrm{NMR}\left(150 \mathrm{MHz}, \mathrm{CD}_{3} \mathrm{OD}\right): \delta 63.52(\mathrm{C}-6), \delta$ $66.53(\mathrm{C}-1), \delta 70.49(\mathrm{C}-4), \delta 71.77(\mathrm{C}-2), \delta 75.50(\mathrm{C}-3), \delta 78.66$ $(\mathrm{C}-5), \delta 108.81\left(\mathrm{C}-2^{\prime}, \mathrm{C}-6^{\prime}\right), \delta 108.95\left(\mathrm{C}-2^{\prime \prime}, 6^{\prime \prime}\right), \delta 119.71\left(\mathrm{Cl}^{\prime}\right)$, $\delta 119.95\left(\mathrm{C}-1^{\prime \prime}\right), \delta 138.45\left(\mathrm{C}-4^{\prime}\right), \delta 138.58\left(\mathrm{C}-4^{\prime \prime}\right), \delta 145.02(\mathrm{C}-$ $\left.3^{\prime}, 5^{\prime}\right), \delta 145.07\left(\mathrm{C}-3^{\prime \prime}, 5^{\prime \prime}\right), \delta 166.46\left(\mathrm{C}-7^{\prime}\right), \delta 167.01\left(\mathrm{C}-7^{\prime \prime \prime}\right)$.

Compound 4 [2,4-Digalloyl-1,5-anhydroglucitol, Maplexin D] (S7-10)

Gray Amorphous Powder. Negative FAB-MS: $m / z 467$ [M$\mathrm{H}]-;{ }^{1} \mathrm{H}-\mathrm{NMR}\left(600 \mathrm{MHz}, \mathrm{CD}_{3} \mathrm{OD}\right): \delta 3.33(1 \mathrm{H}, \mathrm{t}, J=10.8 \mathrm{~Hz}$, $\mathrm{H}-1 \mathrm{~b}) \delta 3.34(1 \mathrm{H}, \mathrm{dd}, J=6,12 \mathrm{~Hz}, \mathrm{H}-6 \mathrm{~b}) \delta 3.38(1 \mathrm{H}, \mathrm{dd}, J=$ $2.4,12 \mathrm{~Hz}, \mathrm{H}-6 \mathrm{a}) 3.46(1 \mathrm{H}, \mathrm{ddd}, J=2.4,5.4,9.6 \mathrm{~Hz}, \mathrm{H}-5) \delta 3.80$ $(1 \mathrm{H}, \mathrm{t}, J=9.0 \mathrm{~Hz}, \mathrm{H}-3) \delta 3.98(1 \mathrm{H}, \mathrm{dd}, J=5.4,10.8 \mathrm{~Hz}, \mathrm{H}-1 \mathrm{a}) \delta$ $4.80(2 \mathrm{H}, \mathrm{m}, \mathrm{H}-2,4) \delta 6.97\left(2 \mathrm{H}, \mathrm{s}, \mathrm{H}-2^{\prime}, 6^{\prime}\right) \delta 6.97(2 \mathrm{H}, \mathrm{s}, \mathrm{H}-$ $\left.2^{\prime \prime}, 6^{\prime \prime}\right) ;{ }^{13} \mathrm{C}-\mathrm{NMR}\left(150 \mathrm{MHz}, \mathrm{CD}_{3} \mathrm{OD}\right): \delta 61.23(\mathrm{C}-6), \delta 66.47$ $(\mathrm{C}-1), \delta 72.00(\mathrm{C}-4), \delta 72.30(\mathrm{C}-2), \delta 72.95(\mathrm{C}-3), \delta 79.94(\mathrm{C}-$ 5), $\delta 109.23\left(\mathrm{C}-2^{\prime}, \mathrm{C}-6^{\prime}\right), \delta 109.27\left(\mathrm{C}-2^{\prime \prime}, 6^{\prime \prime}\right), \delta 119.51\left(\mathrm{Cl}^{\prime}\right), \delta$ $119.73\left(\mathrm{C}-1^{\prime \prime}\right), \delta 138.83\left(\mathrm{C}-4^{\prime}\right), \delta 138.93\left(\mathrm{C}-4^{\prime \prime}\right), \delta 145.81\left(\mathrm{C}-3^{\prime}\right.$, $\left.5^{\prime}\right), \delta 145.83\left(\mathrm{C}-3^{\prime \prime}, 5^{\prime \prime}\right), \delta 165.49\left(\mathrm{C}-7^{\prime}\right), \delta 165.77\left(\mathrm{C}-7^{\prime \prime}\right)$.

Compound 5 [2,4,6-Trigalloyl-1,5-anhydroglucitol, Maplexin E] (S11-14)

Gray Amorphous Powder. Negative FAB-MS: $m / z \quad 619$ [M-H]-; ${ }^{1} \mathrm{H}-\mathrm{NMR}\left(600 \mathrm{MHz}, \mathrm{CD}_{3} \mathrm{OD}\right): \delta 3.45(1 \mathrm{H}, \mathrm{t}, J=$ $11.2 \mathrm{~Hz}, \mathrm{H}-1 \mathrm{~b}) \delta 3.85(1 \mathrm{H}, \mathrm{ddd}, J=2.1,5.1,9.6 \mathrm{~Hz}, \mathrm{H}-5) \delta$ $4.03(1 \mathrm{H}, \mathrm{t}, J=9.3 \mathrm{~Hz}, \mathrm{H}-3) \delta 4.18(1 \mathrm{H}, \mathrm{dd}, J=5.4,11.2 \mathrm{~Hz}$, $\mathrm{H}-1 \mathrm{a}) \delta 4.21(1 \mathrm{H}, \mathrm{dd}, J=5.1,11.7 \mathrm{~Hz}, \mathrm{H}-6 \mathrm{~b}) \delta 4.42(1 \mathrm{H}, \mathrm{dd}$, $J=2.1,11.7 \mathrm{~Hz}, \mathrm{H}-6 \mathrm{a}) \delta 5.02(1 \mathrm{H}, \mathrm{ddd}, J=5.4,9.6,11.2 \mathrm{~Hz}$, 
H-2) $\delta 5.22(1 \mathrm{H}, \mathrm{t}, J=9.6 \mathrm{~Hz}, \mathrm{H}-4) \delta 7.09-7.11(2 \mathrm{H} \times 3$, each s, H-2', $\left.2^{\prime \prime}, 2^{\prime \prime \prime}, 6^{\prime}, 6^{\prime \prime}, 6^{\prime \prime \prime}\right) ;{ }^{13} \mathrm{C}-\mathrm{NMR}\left(150 \mathrm{MHz}, \mathrm{CD}_{3} \mathrm{OD}\right)$ : $\delta 62.7(\mathrm{C}-6), \delta 66.6(\mathrm{C}-1), \delta 71.1(\mathrm{C}-4), \delta 71.8(\mathrm{C}-2), \delta 73.4$ (C-3), $\delta 76.8(\mathrm{C}-5), \delta$ 108.9-109.4 (C-2', $\left.2^{\prime \prime}, 2^{\prime \prime \prime}, 6^{\prime}, 6^{\prime \prime}, 6^{\prime \prime \prime}\right)$, $\delta$ 119.6-119.8 (C-1, $\left.1^{\prime}, 1^{\prime \prime \prime}\right), \delta 138.5-138.7\left(\mathrm{C}-4^{\prime}, 4^{\prime \prime}, 4^{\prime \prime \prime}\right), \delta$ 145.0-145.2 (C-3', $\left.3^{\prime \prime}, 3^{\prime \prime \prime}, 5^{\prime}, 5^{\prime \prime}, 5^{\prime \prime \prime}\right), \delta$ 166.3-166.7 (C-7', $\left.7^{\prime \prime}, 7^{\prime \prime \prime}\right)$.

Compound 6 [Quercetin-3-O-(2" -galloyl)- $\alpha$-L-rhamnopyranoside] $(S 15,16)$

Yellow Amorphous Powder. ${ }^{1} \mathrm{H}-\mathrm{NMR}\left(600 \mathrm{MHz}, \mathrm{CD}_{3} \mathrm{OD}\right): \delta$ $1.03\left(3 \mathrm{H}, \mathrm{d}, J=5.4 \mathrm{~Hz}, \mathrm{H}-6^{\prime \prime}\right) \delta 3.45-3.48\left(2 \mathrm{H}, \mathrm{m}, \mathrm{H}-4^{\prime \prime}, 5^{\prime \prime}\right)$ $\delta 4.01\left(1 \mathrm{H}, \mathrm{dd}, J=3.6,9.0 \mathrm{~Hz}, \mathrm{H}-3^{\prime \prime}\right) \delta 5.49(1 \mathrm{H}, \mathrm{d}, J=1.5 \mathrm{~Hz}$, $\left.\mathrm{H}-1^{\prime \prime}\right) \delta 5.63\left(1 \mathrm{H}, \mathrm{dd}, J=1.5,3.6 \mathrm{~Hz}, \mathrm{H}-2^{\prime \prime}\right) \delta 6.19(1 \mathrm{H}, \mathrm{d}, J=$ $1.8 \mathrm{~Hz}, \mathrm{H}-6) \delta 6.37(1 \mathrm{H}, \mathrm{d}, J=1.8 \mathrm{~Hz}, \mathrm{H}-8) \delta 6.94(1 \mathrm{H}, \mathrm{d}, J=$ $\left.8.4 \mathrm{~Hz}, \mathrm{H}-5^{\prime}\right) \delta 7.17\left(2 \mathrm{H}, \mathrm{s}, \mathrm{H}-2^{\prime \prime \prime}, 6^{\prime \prime \prime}\right) \delta 7.33(1 \mathrm{H}, \mathrm{d}, J=8.4 \mathrm{~Hz}$, $\left.\mathrm{H}-5^{\prime}\right) \delta 7.34\left(1 \mathrm{H}, \mathrm{dd}, J=2.4,8.4 \mathrm{~Hz}, \mathrm{H}-6^{\prime}\right) \delta 7.36(1 \mathrm{H}, \mathrm{d}, J=$ $\left.2.4 \mathrm{~Hz}, \mathrm{H}-2^{\prime}\right) ;{ }^{13} \mathrm{C}-\mathrm{NMR}\left(150 \mathrm{MHz}, \mathrm{CD}_{3} \mathrm{OD}\right): \delta 16.41\left(\mathrm{C}-6^{\prime \prime}\right)$, $\delta 69.29\left(\mathrm{C}-3^{\prime \prime}\right), \delta 70.79\left(\mathrm{C}-5^{\prime \prime}\right), \delta 72.10\left(\mathrm{C}-2^{\prime \prime}\right), \delta 72.36\left(\mathrm{C}-4^{\prime \prime}\right)$, $\delta 93.35(\mathrm{C}-8), \delta 98.46(\mathrm{C}-6), \delta 99.10\left(\mathrm{C}-1^{\prime \prime}\right), \delta 104.45(\mathrm{C}-10)$, $\delta 108.99\left(\mathrm{C}-2^{\prime \prime \prime}, 6^{\prime \prime \prime}\right), \delta 115.10\left(\mathrm{C}-5^{\prime}\right), \delta 115.51\left(\mathrm{C}-2^{\prime}\right), \delta 119.81$ $\left(\mathrm{C}-1^{\prime \prime \prime}\right), \delta 121.41\left(\mathrm{C}-6^{\prime}\right), \delta 121.49\left(\mathrm{C}-1^{\prime}\right), \delta 134.22(\mathrm{C}-3), \delta 138.55$ $\left(\mathrm{C}-4^{\prime \prime \prime}\right), \delta 144.99\left(\mathrm{C}-5^{\prime \prime \prime}\right), \delta 145.02\left(\mathrm{C}-3^{\prime \prime \prime}\right), \delta 148.42\left(\mathrm{C}-4^{\prime}\right), \delta$ 157.07 (C-9), $\delta 157.88$ (C-2), $\delta 161.71$ (C-5), $\delta 164.40$ (C-7), $\delta$ $166.06\left(\mathrm{C}-7^{\prime \prime \prime}\right), \delta 177.95(\mathrm{C}-4)$.

Compound 7 [Kaempferol-3-O-(2" -galloyl)- $\alpha$-L-rhamnopyranoside] $($ S17, 18)

Yellow Amorphous Powder. Negative FAB-MS: $m / z 583$ [M$\mathrm{H}]-;{ }^{1} \mathrm{H}-\mathrm{NMR}\left(600 \mathrm{MHz}, \mathrm{CD}_{3} \mathrm{OD}\right): \delta 1.02(3 \mathrm{H}, \mathrm{d}, J=6.0 \mathrm{~Hz}$, H-6 $\left.{ }^{\prime \prime}\right), \delta 3.44-3.45\left(1 \mathrm{H}, \mathrm{m}, \mathrm{H}-5^{\prime \prime}\right), \delta 3.47(1 \mathrm{H}, \mathrm{t}, J=9.0 \mathrm{~Hz}$, $\left.\mathrm{H}-4^{\prime \prime}\right), \delta 3.99\left(1 \mathrm{H}, \mathrm{dd}, J=3.6,9.0 \mathrm{~Hz}, \mathrm{H}-3^{\prime \prime}\right), \delta 5.48(1 \mathrm{H}, \mathrm{d}, J$ $\left.=1.8 \mathrm{~Hz}, \mathrm{H}-1^{\prime \prime}\right), \delta 5.62\left(1 \mathrm{H}, \mathrm{dd}, J=1.8,3.6 \mathrm{~Hz}, \mathrm{H}-2^{\prime \prime}\right), \delta 6.19$ $(1 \mathrm{H}, \mathrm{d}, J=1.8 \mathrm{~Hz}, \mathrm{H}-6), \delta 6.37(1 \mathrm{H}, \mathrm{d}, J=1.8 \mathrm{~Hz}, \mathrm{H}-8), \delta 6.96$ $\left(2 \mathrm{H}, \mathrm{d}, J=9.0 \mathrm{~Hz}, \mathrm{H}-2^{\prime}, 6^{\prime}\right), \delta 7.07\left(2 \mathrm{H}, \mathrm{s}, \mathrm{H}-2^{\prime \prime \prime}, 6^{\prime \prime \prime}\right), \delta 7.79$ $\left(2 \mathrm{H}, \mathrm{d}, J=9.0 \mathrm{~Hz}, \mathrm{H}-3^{\prime}, 5^{\prime}\right) ;{ }^{13} \mathrm{C}-\mathrm{NMR}\left(150 \mathrm{MHz}, \mathrm{CD}_{3} \mathrm{OD}\right): \delta$ $16.4\left(\mathrm{C}-6^{\prime \prime}\right), \delta 69.3\left(\mathrm{C}-3^{\prime \prime}\right), \delta 70.8\left(\mathrm{C}-5^{\prime \prime}\right), \delta 72.1\left(\mathrm{C}-2^{\prime \prime}\right), \delta 72.3$ $\left(\mathrm{C}-4^{\prime \prime}\right), \delta 93.4(\mathrm{C}-8), \delta 98.5(\mathrm{C}-6), \delta 99.1\left(\mathrm{C}-1^{\prime \prime}\right), \delta 104.5(\mathrm{C}-$ $10), \delta 109.0\left(\mathrm{C}-2^{\prime \prime \prime}, 6^{\prime \prime \prime}\right), \delta 115.2\left(\mathrm{C}-3^{\prime \prime \prime}, 5^{\prime \prime \prime}\right), \delta 119.8\left(\mathrm{C}-1^{\prime \prime \prime}\right), \delta$ $121.1\left(\mathrm{C}-1^{\prime}\right), \delta 130.5\left(\mathrm{C}-2^{\prime}, 6^{\prime}\right), \delta 134.2(\mathrm{C}-3), \delta 138.5\left(\mathrm{C}-4^{\prime \prime \prime}\right), \delta$ $145.0\left(\mathrm{C}-3^{\prime \prime \prime}, 5^{\prime \prime \prime}\right), \delta 157.1(\mathrm{C}-9), \delta 157.8(\mathrm{C}-2), \delta 160.2\left(\mathrm{C}-4^{\prime}\right)$, $\delta 161.7(\mathrm{C}-5), \delta 164.4(\mathrm{C}-7), \delta 166.0\left(\mathrm{C}-7^{\prime \prime \prime}\right), \delta 177.9(\mathrm{C}-4)$.

2.4. Measurement of DPPH Radical Scavenging Activity. Each sample was added to a $0.1 \mathrm{mM}$ DPPH solution in absolute ethanol. After mixing gently and incubating for $30 \mathrm{~min}$ at room temperature, the optical density of the solution was measured at $540 \mathrm{~nm}$ using an ELISA reader (TECAN, Salzburg, Austria). The DPPH radical scavenging activity was calculated as inhibition rate $(\%)=[1-$ (sample optical density/control optical density) $] \times 100$. The $\mathrm{IC}_{50}$ value was defined as the concentration at which $50 \%$ of the DPPH free radicals were scavenged. Ascorbic acid was used as a positive control.

2.5. Measurement of NBT/Superoxide Scavenging Activity. Each sample was added to a substrate mixture containing
EDTA (0.05 mM), hypoxanthine $(0.2 \mathrm{mM})$, and nitroblue tetrazolium (NBT, $1 \mathrm{mM})$ in $50 \mathrm{mM}$ phosphate buffer $(\mathrm{pH}$ 7.5). The reaction was then initiated by the addition of xanthine oxidase $(1.2 \mathrm{U} / \mu \mathrm{L}$, Sigma). The NBT/superoxide radical scavenging activity was calculated as inhibition rate $(\%)=[1-($ sample optical density/control optical density) $]$ $\times 100$. The $\mathrm{IC}_{50}$ value was defined as the concentration at which $50 \%$ of the NBT/superoxide radicals were scavenged. Allopurinol (Sigma) was used as a positive control.

2.6. Measurement of Inhibition of NO Production. RAW264.7 mouse macrophage cells were seeded onto 96 -well plates $(3 \times$ $10^{4}$ cells/well) and incubated for $2 \mathrm{~h}$ at $37^{\circ} \mathrm{C}$ in a humidified atmosphere $\left(5 \% \mathrm{CO}_{2}\right)$. The cells were then incubated in medium containing $1 \mu \mathrm{g} / \mathrm{mL}$ lipopolysaccharide (LPS, Sigma) and the sample of interest. After incubating for an additional $24 \mathrm{~h}$, Griess reagent (0.1\% naphthylethylenediamine and $1 \%$ sulfanilamide in $5 \% \mathrm{H}_{3} \mathrm{PO}_{4}$ solution, Sigma) was mixed with the culture supernatant. The absorbance of the samples was then measured at $540 \mathrm{~nm}$ using a microplate reader, and the amount of nitrite in each sample was calculated from a sodium nitrite standard curve. The inhibition rate of NO production was calculated as inhibition rate $(\%)=[1-$ (sample optical density - blank optical density)/(control optical density - blank optical density) $] \times 100$. The $\mathrm{IC}_{50}$ value was defined as the concentration at which the nitrite radicals were reduced by $50 \%$. L-NMMA was used as a positive control.

2.7. Induction of Diabetes and Experimental Design. Male Sprague-Dawley rats (130-150 g each) were housed in the departmental animal facility for two weeks before the experiment. Environmental conditions included a temperature of $22-24^{\circ} \mathrm{C}$, a $12 \mathrm{~h}$ day/ $12 \mathrm{~h}$ night cycle, and relative air humidity of $40-60 \%$. The animals were provided with standard rodent diet and water ad libitum and were divided into 4 groups ( $n=6$ per group). For the induction of experimental diabetes, $45 \mathrm{mg} / \mathrm{kg}$ b.w. ( $0.1 \mathrm{M}$ citrate buffer of $\mathrm{pH} 4.5$ in a volume of $1 \mathrm{~mL} / \mathrm{kg}$ ) of STZ (Sigma, USA) was injected intraperitoneally. Mice with a blood glucose level greater than $300 \mathrm{mg} / \mathrm{dL}$ were considered to be diabetic and were included in the experiment. The 24 rats were divided into the following 4 groups: Group I, normal control; Group II, diabetic control; Group III, diabetic rats treated daily with AG extract (500 mg/kg b.w.). Group IV, diabetic rats treated daily with 3 (AT, $100 \mathrm{mg} / \mathrm{kg}$ b.w.), respectively.

2.8. Biochemical Analysis. Rats were fasted overnight, after which blood samples were collected from the inferior vena cava. Blood glucose levels were detected using a portable blood glucose meter (Roche Diagnostics GmbH, Germany). GOT and GPT levels were determined using commercial kits (Asan Pharmaceutical, Republic of Korea).

2.9. Statistical Analysis. The DPPH, NBT/superoxide, and nitrite radical scavenging activities of each compound were analyzed by one-way analysis of variance (ANOVA) followed by the Student-Newman-Keuls (SNK) post hoc test. The body 
<smiles>O=C(O)c1cc(O)c(O)c(O)c1</smiles><smiles>[R20]C[C@H]1OC[C@@H]([R20])[C@H](O)[C@@H]1[R6]</smiles>

\begin{tabular}{cccc}
\hline & $\mathrm{R}_{1}$ & $\mathrm{R}_{2}$ & $\mathrm{R}_{3}$ \\
\hline $\mathbf{2}$ & $\mathrm{G}$ & $\mathrm{H}$ & $\mathrm{H}$ \\
$\mathbf{3}$ & $\mathrm{G}$ & $\mathrm{G}$ & $\mathrm{H}$ \\
$\mathbf{4}$ & $\mathrm{H}$ & $\mathrm{G}$ & $\mathrm{G}$ \\
$\mathbf{5}$ & $\mathrm{G}$ & $\mathrm{G}$ & $\mathrm{G}$ \\
\hline \multicolumn{4}{l}{$\mathrm{G}=$ galloyl } \\
\end{tabular}<smiles>[R]c1cc(-c2oc3cc(O)cc(O)c3c(=O)c2O[C@@H]2O[C@H](C)[C@@H](O)[C@H](O)[C@H]2OC(=O)c2cc(O)c(O)c(O)c2)ccc1O</smiles>

FIgURE 1: Structures of compounds 1-7 from AG.

weight, blood glucose, and GOT/GPT levels of the rats were analyzed by the Mann-Whitney $U$ test.

\section{Results and Discussion}

3.1. Isolation and Structural Identification. The repeated chromatographic isolation of the $80 \% \mathrm{MeOH}$ extract of AG yielded seven galloyl derivatives: gallic acid (1) [8], ginnalin $\mathrm{B}(2)[8,18]$, acertannin $(3)[8,17]$, maplexin D (4) [20], maplexin E (5) [20], quercetin-3-O-(2" -galloyl)$\alpha$-L-rhamnopyranoside (6) [21], and kaempferol-3-O- $\left(2^{\prime \prime}\right.$ galloyl)- $\alpha$-L-rhamnopyranoside (7) [21] (Figure 1). All isolated compounds were identified by comparing the spectral data with values reported in the literature. This study is the first to report the isolation of compounds 4 and 5 from AG.

\subsection{Evaluation of Antioxidative and Anti-Inflammatory Activ-} ities. To assess the antioxidative activities of the compounds isolated from AG, the DPPH radical scavenging ability of each compound was measured. All compounds exhibited potent antioxidative activities, as shown in Table 1. Of particular note, compounds 3-5 exhibited significantly more potent scavenging activity than the positive control (ascorbic acid) $(p<0.05)$. These gallotannins (3-5) all have more than two galloyl groups, which are known to eliminate free radicals. Moreover, the extent of the DPPH radical scavenging activity depended on the number of galloyl groups $(\mathbf{1}, \mathbf{2}<\mathbf{3}$, 4, 5). Flavonoids are also well-known natural antioxidants. In particular, flavonoids with a keto-enol moiety at positions C-4 and C-5 and phenolic hydroxyl groups in the B-ring are known to act as crucial electron acceptors. Moreover, compounds 6 and 7 possessed an additional galloyl moiety at the O-2 position of the rhamnopyranoside ring. We found that the DPPH radical scavenging activity of compound $\mathbf{6}$, which possessed a $3^{\prime}, 4^{\prime}$-dihydroxyphenyl B-ring, was more potent than that of compound 7 , which possessed a $4^{\prime}$ hydroxyphenyl B-ring. Moreover, this effect was significantly greater than that of ascorbic acid $(p<0.05)$ (Table 1$)$.

The enzyme xanthine oxidase catalyzes the oxidation of hypoxanthine and xanthine to uric acid, producing a superoxide radical and hydrogen peroxide. Thus, the superoxide radical scavenging activities of the compounds isolated from AG were evaluated using a colorimetric NBT reduction assay. Similar to the DPPH radical scavenging results, compounds 3-5 scavenged superoxide radicals more effectively than compounds 1 and 2, indicating that the number of galloyl 
TABLE 1: IC $_{50}$ values of compounds 1-7 for DPPH scavenging, NBT/superoxide radical scavenging, and inhibition of nitric oxide production by LPS-stimulated RAW264.7 cells.

\begin{tabular}{lccc}
\hline Compound & DPPH radical scavenging & $\mathrm{IC}_{50}(\mu \mathrm{M})^{\mathrm{a}}$ \\
activity & $\begin{array}{c}\text { NBT/superoxide } \\
\text { scavenging activity }\end{array}$ & $\begin{array}{c}\text { Inhibition of NO } \\
\text { production }\end{array}$ & $100<$ \\
\hline $\mathbf{1}$ & $10.44 \pm 0.77^{\mathrm{b}, \mathrm{c}}$ & $17.08 \pm 1.33^{\mathrm{e}}$ & $100<$ \\
$\mathbf{3}$ & $12.14 \pm 0.03^{\mathrm{c}}$ & $20.80 \pm 0.84^{\mathrm{e}}$ & $100<$ \\
$\mathbf{4}$ & $6.87 \pm 1.05^{\mathrm{a}}$ & $2.96 \pm 0.14^{\mathrm{a}}$ & $100<$ \\
$\mathbf{5}$ & $6.92 \pm 0.52^{\mathrm{a}}$ & $3.01 \pm 0.12^{\mathrm{a}}$ & $36.08 \pm 2.12^{\mathrm{b}}$ \\
$\mathbf{6}$ & $5.72 \pm 0.30^{\mathrm{a}}$ & $2.83 \pm 0.09^{\mathrm{a}}$ & $76.46 \pm 4.68^{\mathrm{c}}$ \\
7 & $12.44 \pm 0.30^{\mathrm{c}}$ & $5.20 \pm 0.57^{\mathrm{b}}$ & $35.62 \pm 1.25^{\mathrm{b}}$ \\
Vitamin C & $18.92 \pm 0.33^{\mathrm{d}}$ & $12.44 \pm 1.54^{\mathrm{c}, \mathrm{d}}$ & - \\
Allopurinol & $13.11 \pm 0.12^{\mathrm{c}}$ & - & - \\
L-NMMA & - & $9.75 \pm 1.22^{\mathrm{c}}$ & - \\
\hline
\end{tabular}

${ }^{\mathrm{a}}$ Values are presented as mean $\pm \mathrm{SD}(n=3)$.

Different superscript letters indicate a significant difference $(p$ value $<0.05$ ).

groups is crucial for the elimination of superoxide radicals $(p<0.05)$ (Table 1). Flavonoids are also well-known natural xanthine oxidase inhibitors and superoxide radical scavengers. The hydroxyl groups at positions C-5/C-7 and the double bond between positions C-2 and C-3 have both been shown to be essential for effective inhibition of xanthine oxidase, while the hydroxyl group at position $\mathrm{C}-3^{\prime}$ has been reported to influence superoxide radical scavenging [22]. With respect to these structure-activity relationships, compounds 6 and 7 (flavonols) showed either the same or significantly more potent scavenging activity than the positive control, allopurinol $(p<0.05)$ (Table 1). Thus, these results indicate that other bioavailable galloyl derivatives such as oligo-galloyl-substituted acer-tannins or galloyl flavonoids from AG contribute more to the elimination of intracellular oxidative stress compared to compound 2.

Next, we confirmed the inhibitory effects of the compounds isolated from AG on nitric oxide production in LPSstimulated RAW264.7 cells. Macrophages produce various proinflammatory mediators, including the short-lived free radical NO. Moreover, lipopolysaccharide (LPS), a component of the cell wall of Gram-negative bacteria, is one of the most powerful activators of macrophages and stimulates NO production. Of the gallotannins, only compound 5 (a trigalloyl-substituted tannin) inhibited nitric oxide production; in contrast, treatment with compounds 1-4 did not have any significant effect on NO production. We hypothesize that compounds 1-4 were too hydrophilic to reduce intracellular NO production (Table 1). The $x \log P$ scores of compounds 1-4, as provided by PubChem, were all negative. Consistent with this tendency, the inhibitory activity of compound 7 , which possessed a $4^{\prime}$-hydroxyphenyl B-ring, was more potent than that of compound $\mathbf{6}$, which possessed a $3^{\prime}, 4^{\prime}$-dihydroxyphenyl B-ring $(p<0.05)$ (Table 1$)$ [19]. Of particular note, trigalloyl-substituted tannins and kaempferol-derived flavonoids have been reported to reduce iNOS expression via NF-kB inactivation [23, 24].
3.3. Evaluation of Antidiabetic Activity. Streptozotocin is widely applied to induce experimental diabetes due to its ability to selectively target and destroy insulin-producing pancreatic islet beta cells [25]. In the present study, we induced experimental diabetes through IP injection of STZ and evaluated the antidiabetic effect of AG via oral administration of AG extract $(500 \mathrm{mg} / \mathrm{kg}$ b.w). Alternatively, we administered the major compound of AG, ginnalin A (3) $(100 \mathrm{mg} / \mathrm{kg} \mathrm{b.w})$. The STZ IP injection group exhibited significantly elevated fasting blood glucose and serum GOT/GPT levels compared to the normal control group. Oral administration of AG extract significantly reduced these increases in fasting blood glucose and serum GOT/GPT levels compared to the diabetic control group. However, treatment group of $\mathbf{3}$ did not exhibit any significant changes, even though the median values were decreased. In addition, STZ injection resulted in a significant decrease in whole body weight, which was restored by the oral administration of AG. Similar to above, this rescue was not observed in treatment group of $\mathbf{3}$ (Figure 2).

Diabetes is a pathological process related to an unbalanced production of ROS, such as hydroxyl radicals $\left({ }^{\circ} \mathrm{OH}\right)$, superoxide anions $\left(\mathrm{O}_{2}\right)$, and $\mathrm{H}_{2} \mathrm{O}_{2}$, and RNS such as nitric oxide (NO) and peroxynitrite $\left(\mathrm{ONOO}^{-}\right)$[26]. Excessive oxidative stress elevates aldose reductase activity and stimulates the hexosamine biosynthetic pathway, as well as increasing formation of advanced glycation end-products [27]. In addition, lipid peroxide production and abnormal immune responses result in various complications such as diabetic retinopathy, nephropathy, neuropathy, and atherosclerosis [28]. Thus, the application of natural antioxidants that offer resistance against oxidative stress to diabetes has been a topic of interest.

STZ has been reported to generate robust production of nitric oxide and hydroxyl radicals or ROS [29], leading to inflammation and apoptosis of $\beta$-cells in the pancreas and thereby mimicking autoimmune diabetes. Moreover, at high concentrations, acute toxicity in extrapancreatic organs such 

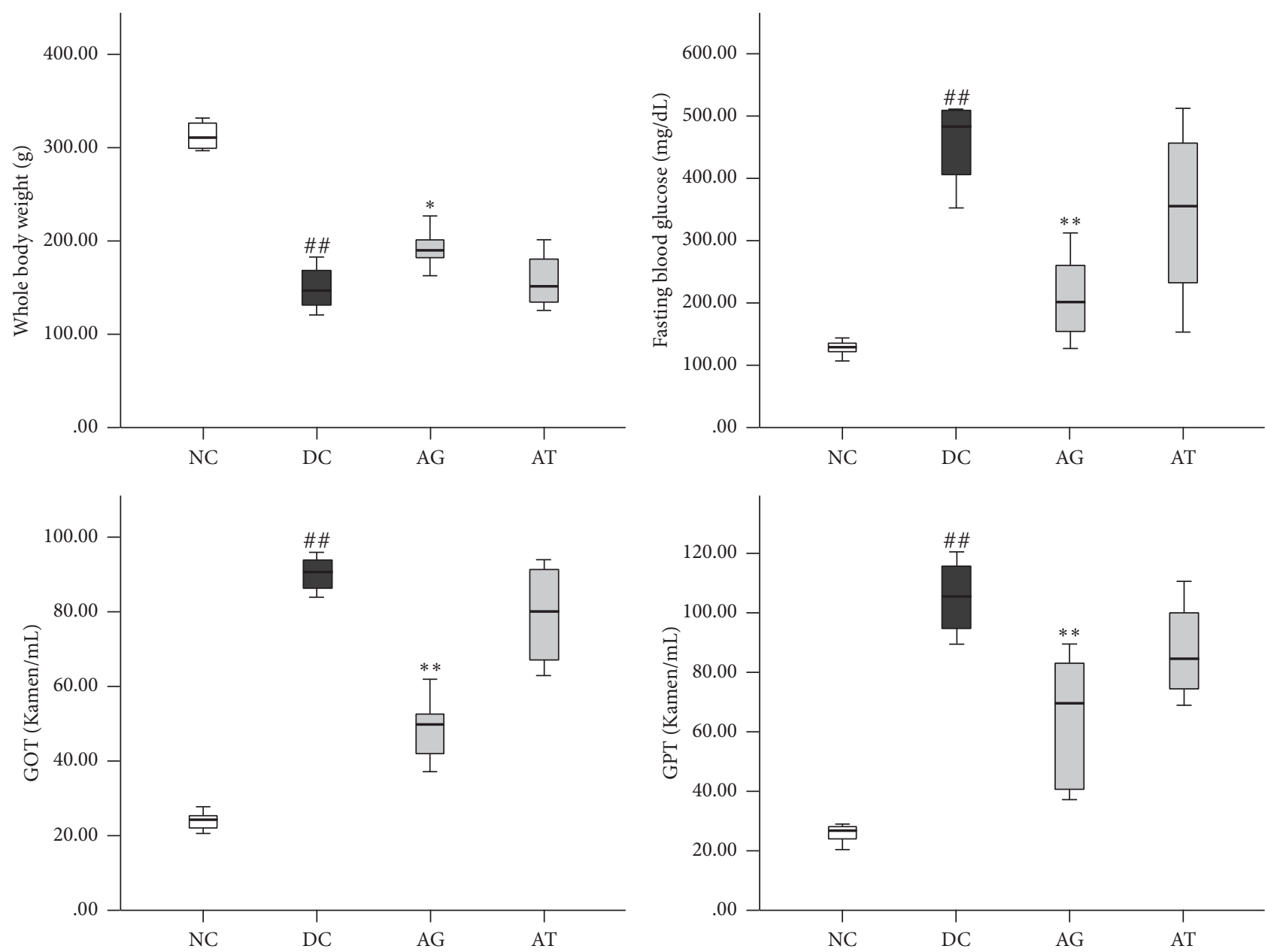

FIGURE 2: Effects of AG and AT (acertannin) on whole body weight, fasting blood glucose, and GOT and GPT levels in STZ-induced diabetic rats. The experimental diabetic rats were orally given AG $(500 \mathrm{mg} / \mathrm{kg}$ b.w.) or AT ( $100 \mathrm{mg} / \mathrm{kg}$ b.w.) for 2 weeks $(n=6$ per group). Results are expressed as median and 75 th $/ 25$ th percentiles $\left({ }^{*} p<0.05\right.$ and ${ }^{* *} p<0.01$ versus diabetic control group; ${ }^{\# \#} p<0.01$ versus normal control group).

as the liver has been reported [30]. Oral administration of AG to diabetic rats effectively recovered fasting blood glucose and serum GOT/GPT levels. These results suggest that AG has a protective effect against oxidative stress in STZ-induced diabetic rats.

In contrast, acertannin (3), the main crystalline tannin of AG, did not show significant antidiabetic activity. Consistent with this result, $\mathbf{3}$ and the monogalloyl- and digalloylsubstituted tannins $(\mathbf{1}, \mathbf{2}$, and $\mathbf{4})$ did not have any effect on NO production in LPS-stimulated RAW264.7 cells.

$\alpha$-Glucosidase secreted from intestinal chorionic epithelium is a carbohydrate hydrolase. $\alpha$-Glucosidase (EC 3.2.1.20) inhibitors, as a new antidiabetic drug from 1980s, slow down the process of digestion and absorption of carbohydrates by competitively blocking the glucosidase activity. A previous report [31] found that galloyl substitution (more than three substitutions at the 1,5-anhydroglucitol core) resulted in potent inhibition of $\alpha$-glucosidase, with a galloyl group at C- 6 being essential for this activity. In particular, the $\alpha$ glucosidase inhibitory activity of $3\left(\mathrm{IC}_{50}=88.42 \pm 6.94 \mu \mathrm{M}\right)$ was reported to be 10 times less than that of $\mathbf{5}\left(\mathrm{IC}_{50}=\right.$ $8.26 \pm 0.37 \mu \mathrm{M}$ ) [31]. Moreover, flavonoid hydroxylation and galloylation have been reported to improve the inhibitory activity of $\alpha$-glucosidase [32]. This finding suggests that oligogalloyl-substituted acer-tannins or galloyl flavonoids from AG also directly influence glucose metabolism. Future studies will aim to elucidate the active constituents of AG and to elucidate the mechanism of action of this extract.

\section{Conclusion}

Chromatographic isolation of the $80 \% \mathrm{MeOH}$ extract of Acer ginnala (AG) yielded seven galloyl derivatives: gallic acid (1), ginnalin B (2), acertannin (3), maplexin $\mathrm{D}$ (4), maplexin $\mathrm{E}$ (5), quercetin-3-O-(2" -galloyl)- $\alpha$-Lrhamnopyranoside (6), and kaempferol-3-O-(2" -galloyl $)-\alpha-$ L-rhamnopyranoside (7). Compounds 3-7 exhibited particularly potent radical scavenging activities, and $\mathbf{5}$ and $\mathbf{7}$ strongly inhibited nitric oxide production in LPS-stimulated RAW264.7 cells. In addition, oral administration of AG 
extract $(500 \mathrm{mg} / \mathrm{kg}$ b.w.) for 2 weeks resulted in significantly lower fasting blood glucose and serum GOT/GPT levels in STZ-induced experimental diabetic rats. These results suggest that the leaves of AG could be useful in the treatment of diabetes mellitus. However, identification of the active constituents of AG responsible for its antidiabetic activity will require further in vivo studies. Such studies could be designed based on reports that acertannin exhibits antidiabetic activity in vitro and in the acute glucose tolerance test in vivo.

\section{Competing Interests}

The authors declare that there is no conflict of interests regarding the publication of this paper.

\section{Authors' Contributions}

Kwan Hee Park and Kyu Hyeong Yoon contributed equally to this work.

\section{Acknowledgments}

This study was supported by the Basic Science Research Program through the National Research Foundation of Korea (NRF-2016R1D1A1B03930307), which is funded by the Ministry of Education, Science, and Technology.

\section{References}

[1] World Health Organization, Global Report on Diabetes, WHO, Geneva, Switzerland, 2016.

[2] C. D. Mathers and D. Loncar, "Projections of global mortality and burden of disease from 2002 to 2030," PLoS Medicine, vol. 3, no. 11, article e442, 2006.

[3] WHO, "Definition, diagnosis and classification of diabetes mellitus and its complications. Part 1: diagnosis and classification of diabetes mellitus," Tech. Rep. WHO/NCD/NCS/99.2, World Health Organization, Geneva, Switzerland, 1999.

[4] T. Inoguchi, P. Li, F. Umeda et al., "High glucose level and free fatty acid stimulate reactive oxygen species production through protein kinase $\mathrm{C}$-dependent activation of $\mathrm{NAD}(\mathrm{P}) \mathrm{H}$ oxidase in cultured vascular cells," Diabetes, vol. 49, no. 11, pp. 1939-1945, 2000.

[5] M. Crook, "Type 2 diabetes mellitus: a disease of the innate immune system? An update," Diabetic Medicine, vol. 21, no. 3, pp. 203-207, 2004.

[6] D. M. Van Gelderen, P. C. De Jong, and H. J. Oterdom, Maples of the World, Timber Press, Portland, Ore, USA, 1994.

[7] T. Arnason, R. J. Hebda, and T. Johns, "Use of plants for food and medicine by Native Peoples of eastern Canada," Canadian Journal of Botany, vol. 59, no. 11, pp. 2189-2325, 1981.

[8] S. E. Choi, K. H. Park, M. H. Oh et al., "Antioxidative activities and quantitative determination of gallotannins from barks of Acer ginnala Maxim," Korean Journal of Pharmacognosy, vol. 41, no. 3, pp. 174-179, 2010.

[9] A. G. Perkin and Y. Uyeda, "XIII.-Occurrence of a crystalline tannin in the leaves of the Acer ginnala," Journal of the Chemical Society, Transactions, vol. 121, pp. 66-76, 1922.
[10] K. Bock, N. Faurschou laCour, S. R. Jensen, and B. J. Nielsen, "The structure of acertannin," Phytochemistry, vol. 19, no. 9, p. 2033, 1980.

[11] Y. K. Son and Y. N. Han, "Isolation of triterpenoid saponins from the stems of Acer ginnala Maxim," Korean Journal of Pharmacognosy, vol. 33, no. 4, pp. 301-304, 2002.

[12] R.-L. Lu, F.-L. Hu, and T. Xia, "Activity-guided isolation and identification of radical scavenging components in Gao-Cha tea," Journal of Food Science, vol. 75, no. 8, pp. H239-H243, 2010.

[13] S. S. Han, S. C. Lo, Y. W. Choi, J. H. Kim, and S. H. Baek, "Antioxidant activity of crude extract and pure compounds of Acer ginnala Max," Bulletin of the Korean Chemical Society, vol. 25, no. 3, pp. 389-391, 2004.

[14] H. C. Yong, S. H. Sung, O. L. Hyun, and H. B. Seung, "Biological activity of bioactive components from Acer ginnala Max," Bulletin of the Korean Chemical Society, vol. 26, no. 9, pp. 1450$1452,2005$.

[15] N. Nagai, Y. Ito, and A. Taga, "Comparison of the enhancement of plasma glucose levels in type 2 diabetes Otsuka Long-Evans Tokushima Fatty rats by oral administration of sucrose or maple syrup," Journal of Oleo Science, vol. 62, no. 9, pp. 737-743, 2013.

[16] Y. M. Lee, Y. S. Kim, J. H. Kim, and J. S. Kim, "Screening of Korean herbal medicines with inhibitory activity on advanced glycation end products formation (IX)," Korean Journal of Pharmacognosy, vol. 44, no. 3, pp. 298-304, 2013.

[17] A. Honma, T. Koyama, and K. Yazawa, "Anti-hyperglycemic effects of sugar maple Acer saccharum and its constituent acertannin," Food Chemistry, vol. 123, no. 2, pp. 390-394, 2010.

[18] A. Honma, T. Koyama, and K. Yazawa, "Anti-hyperglycaemic effects of the Japanese red maple Acer pycnanthum and its constituents the ginnalins B and C," Journal of Enzyme Inhibition and Medicinal Chemistry, vol. 26, no. 2, pp. 176-180, 2011.

[19] W. Bi, Y. Gao, J. Shen et al., "Traditional uses, phytochemistry, and pharmacology of the genus Acer(maple): a review," Journal of Ethnopharmacology, vol. 189, pp. 31-60, 2016.

[20] C. Wan, T. Yuan, L. Li et al., "Maplexins, new $\alpha$-glucosidase inhibitors from red maple (Acer rubrum) stems," Bioorganic and Medicinal Chemistry Letters, vol. 22, no. 1, pp. 597-600, 2012.

[21] Y. Kim, D.-S. Jang, S.-H. Park et al., "Flavonol glycoside gallate and ferulate esters from Persicaria lapathifolia as inhibitors of superoxide production in human monocytes stimulated by unopsonized zymosan," Planta Medica, vol. 66, pp. 72-74, 2000.

[22] P. Cos, L. Ying, M. Calomme et al., "Structure-activity relationship and classification of flavonoids as inhibitors of xanthine oxidase and superoxide scavengers," Journal of Natural Products, vol. 61, no. 1, pp. 71-76, 1998.

[23] V. García-Mediavilla, I. Crespo, P. S. Collado et al., "The anti-inflammatory flavones quercetin and kaempferol cause inhibition of inducible nitric oxide synthase, cyclooxygenase2 and reactive C-protein, and down-regulation of the nuclear factor kappaB pathway in Chang Liver cells," European Journal of Pharmacology, vol. 557, no. 2-3, pp. 221-229, 2007.

[24] M.-S. Kim, S.-B. Park, K. Suk et al., "Gallotannin isolated from euphorbia species, 1,2,6-Tri-O-galloyl- $\beta$-D-allose, decreases nitric oxide production through inhibition of nuclear factor$\kappa>\mathrm{B}$ and downstream inducible nitric oxide synthase expression in macrophages," Biological and Pharmaceutical Bulletin, vol. 32, no. 6, pp. 1053-1056, 2009.

[25] E. B. Johansson and H. Tjalve, "Studies on the tissue-deposition and fate of [14C] streptozotocin with special reference to the pancreatic islets," Acta Endocrinologica, vol. 89, pp. 339-351, 1978. 
[26] P. Subash-Babu, A. A. Alshatwi, and S. Ignacimuthu, "Beneficial antioxidative and antiperoxidative effect of cinnamaldehyde protect streptozotocin-induced pancreatic $\beta$-cells damage in wistar rats," Biomolecules and Therapeutics, vol. 22, no. 1, pp. 4754, 2014.

[27] M. C. Eppens, M. E. Craig, J. Cusumano et al., "Prevalence of diabetes complications in adolescents with type 2 compared with type 1 diabetes," Diabetes Care, vol. 29, no. 6, pp.1300-1306, 2006.

[28] V. Granberg, N. Ejskjaer, M. Peakman, and G. Sundkvist, "Autoantibodies to autonomic nerves associated with cardiac and peripheral autonomic neuropathy," Diabetes Care, vol. 28, no. 8, pp. 1959-1964, 2005.

[29] N. S. Kwon, S. H. Lee, C. S. Choi, T. Kho, and H. S. Lee, "Nitric oxide generation from streptozotocin," FASEB Journal, vol. 8, no. 8, pp. 529-533, 1994.

[30] R. H. Bell Jr. and R. J. Hye, "Animal models of diabetes mellitus: physiology and pathology," Journal of Surgical Research, vol. 35, no. 5, pp. 433-460, 1983.

[31] Z. Yin, W. Zhang, F. Feng, Y. Zhang, and W. Kang, " $\alpha-$ Glucosidase inhibitors isolated from medicinal plants," Food Science and Human Wellness, vol. 3, no. 3-4, pp. 136-174, 2014.

[32] J. Xiao, G. Kai, K. Yamamoto, and X. Chen, "Advance in dietary polyphenols as $\alpha$-glucosidases inhibitors: a review on structureactivity relationship aspect," Critical Reviews in Food Science and Nutrition, vol. 53, no. 8, pp. 818-836, 2013. 


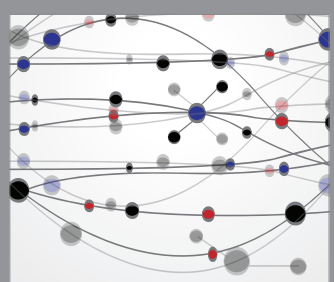

The Scientific World Journal
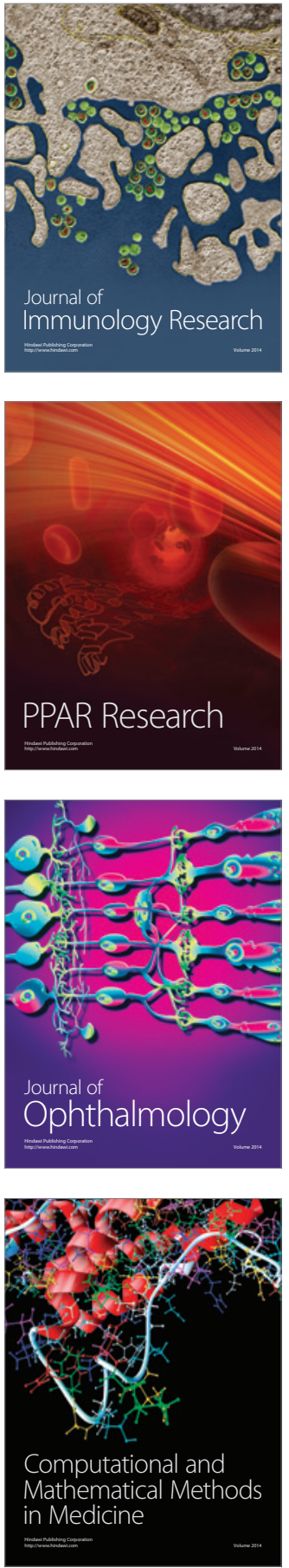

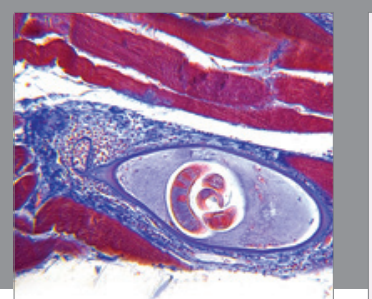

Gastroenterology Research and Practice
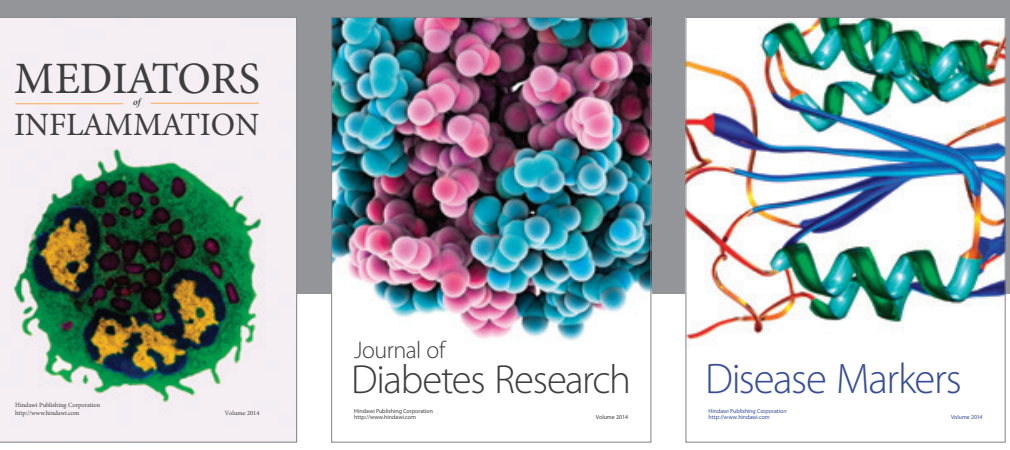

Disease Markers

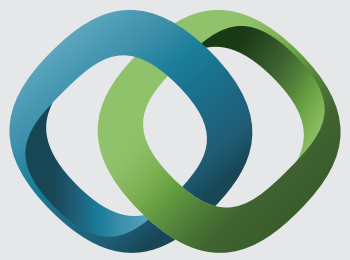

\section{Hindawi}

Submit your manuscripts at

https://www.hindawi.com
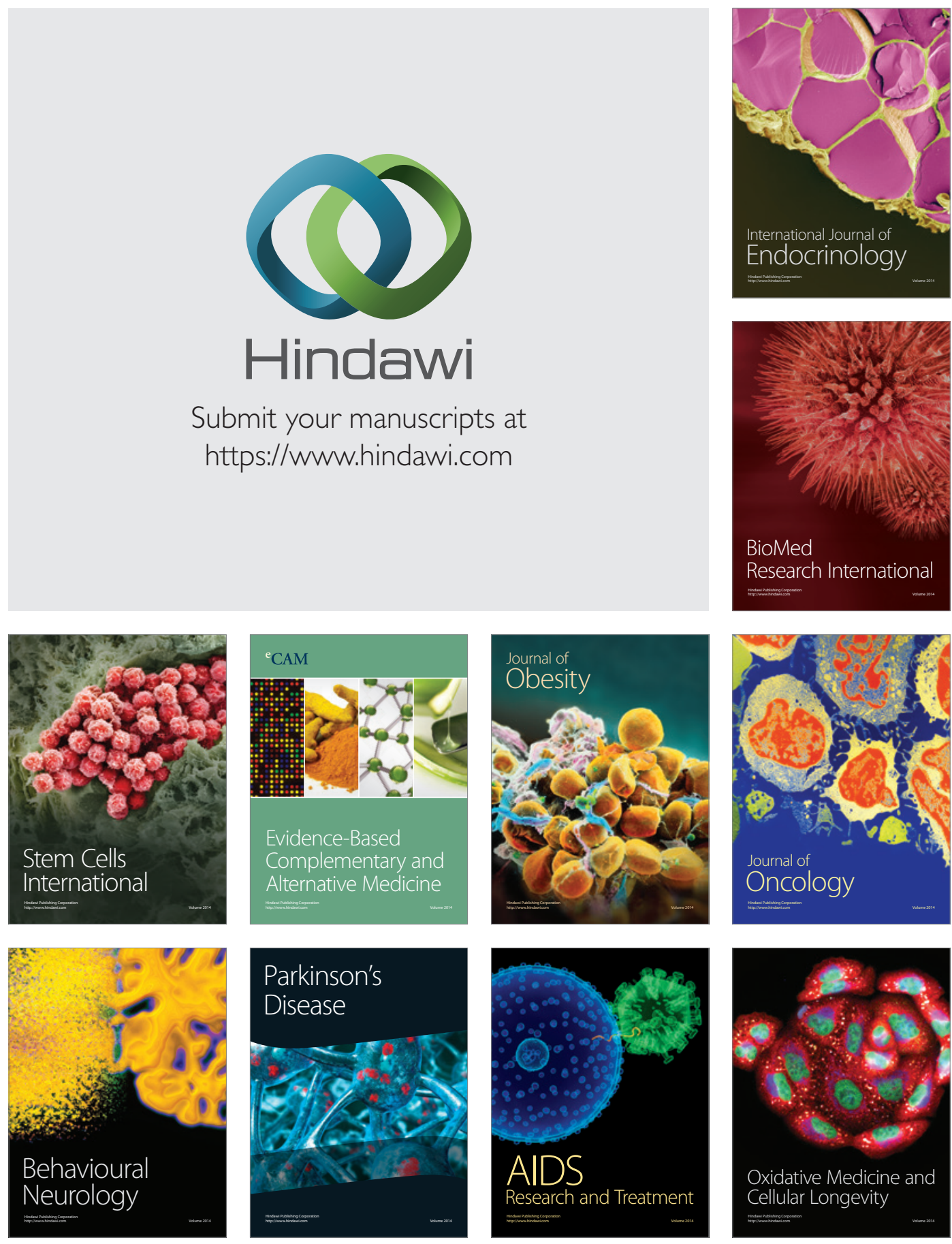